ENCYCLOPEDDIE Encyclopédie berbère

BERBERE

$36 \mid 2013$

36| Oryx - Ozoutae

\title{
Os / IYass (ethnolinguistique)
}

Salem Chaker

\section{OpenEdition}

Journals

Édition électronique

URL : https://journals.openedition.org/encyclopedieberbere/2840

DOI : $10.4000 /$ encyclopedieberbere. 2840

ISSN : 2262-7197

\section{Éditeur}

Peeters Publishers

\section{Édition imprimée}

Date de publication : 2 juin 2013

Pagination : 5881-5884

ISBN : 978-2-7584-0194-0

ISSN : 1015-7344

\section{Référence électronique}

Salem Chaker, « Os / I yəss (ethnolinguistique) », Encyclopédie berbère [En ligne], 36 | 2013, document 033, mis en ligne le 12 mars 2021, consulté le 17 février 2022. URL : http://journals.openedition.org/ encyclopedieberbere/2840; DOI : https://doi.org/10.4000/encyclopedieberbere.2840

Ce document a été généré automatiquement le 17 février 2022.

(c) Tous droits réservés 


\section{Os / I zass (ethnolinguistique)}

\section{Salem Chaker}

1 Le mot "os ", iłəss en berbère ${ }^{1}$, présente un grand intérêt linguistique, ethnolinguistique et anthropologique. Il fait partie de ces termes fondamentaux de l'anatomie (humaine, animale et végétale) caractérisés par une remarquable unité à travers l'ensemble du domaine berbère :

- Kabyle : izəss, plur. izsan (Dallet, p. 629).

- Chaoui : iys, plur. iysan (Huyghe, p. 462).

- Chenoua : ixəs, plur. iysan (Laoust, p. 139).

- Chleuh : ixss, plur. ixsan (Destaing, p. 206) ; iys, plur. iðsan (Laoust, p. 36 ; Jordan, p. 75).

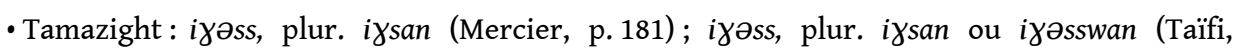
p. 207-8).

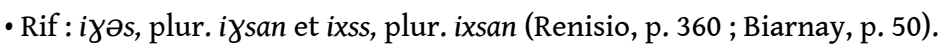

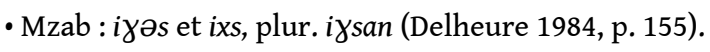

- Ouargla : iðəs, plur. izəsan ou ixsan (Delheure 1987, p. 246).

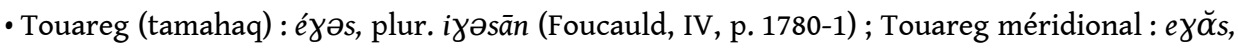
plur. izəsān (Prasse p. 305).

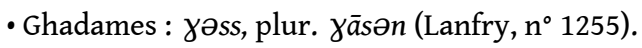

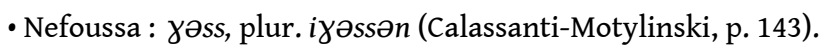

- Zenaga : ?ss (Taine-Cheikh, p. 52).

2

Il présente d'ailleurs des correspondances assez nettes avec des formes lexicales sémitiques (arabe), égyptiennes et tchadiques (cf. Prasse et al. 2002, p. 305) et appartient donc certainement au fonds lexical commun afro-asiatique.

4 Le terme entre dans la formation des composés lexicaux les plus anciens et les plus communs à l'ensemble berbère :

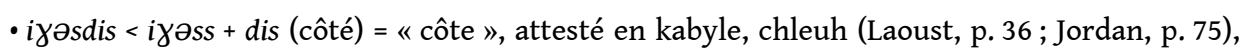
en rifain (Renisio, p. 360), en tamazight (Taïfi, p. 208)...

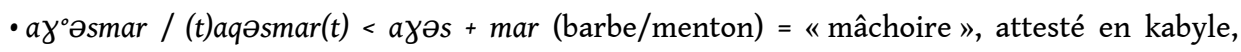
Chenoua (Laoust, p. 139), en chleuh (Laoust, p. 32); en rifain (Renisio, p. 360 ; Biarnay, p. 50)... 
- aqesbud < aqəs + bud (tronc, ventre, nombril...) = « gigot, cuisse », kabyle (Dallet, p. 682-3).

forme de leur second élément, ces composés anciens confirment l'abse ancienne de la marque nominale initiale ( $a-/ t a-;$ cf. N62 « Nom/Nominal », EB XXXIV) et le lien étroit qui unit [8] et [q] dans le système phonologique fondamental du berbère commun, où ces deux sons ne devaient être que des variantes (régionales et/ou contextuelles) d'un seul phonème. De même, le couple iðəs(s)/ixs(s) confirme que la constrictive vélaire sourde $[\mathrm{x}]$ est - quand elle n'est pas empruntée à l'arabe - toujours secondaire en berbère et résulte de l'assourdissement de $[\gamma]$ lorsque ce dernier entre en contact direct avec une sourde, consécutivement à la disparation d'un élément

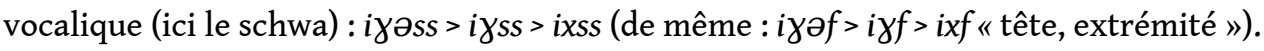
Une forme féminine apparentée, tayəssa, désigne "l'ensemble des os », « le squelette », «l'ossature », la « carcasse » (touareg: Foucauld, IV, p. 1781).

8 Les différentes formes attestées, notamment le pluriel en -an (avec voyelle longue [ān]), la présence de la labio-vélarisation dans $a \gamma^{w} \partial s m a r$, et la tension nette de la sifflante finale au singulier dans la plupart des attestations synchroniques doivent être interprétées comme la trace d'une ancienne consonne finale disparue, présentant sans doute le trait de labialité (=/w/ ?) : on posera donc une racine primitive * $\mathrm{rS}(\mathrm{W}) / \mathrm{QS}(\mathrm{W})^{2}$.

9 Au plan sémantique, l'intérêt de ce mot n'est pas moins grand, puisqu'il désigne à la fois «l'os» et le «noyau » d'un fruit, qui sont donc associés comme «composant interne dur d'un animal, être humain ou d'un végétal »; parfois même la " carapace » (Chenoua), la «charpente» (touareg)... Le sens «noyau» est certainement fondamental car très largement attesté à travers le domaine berbère (kabyle, tamazight, Mzab, Ouargla, Ghadamès, touareg méridional, zenaga...) et peut donc difficilement être considéré comme secondaire contrairement à ce qu'écrit Prasse (2002, p. 305 : « par extension »).

10 Le terme désigne aussi dans la plupart des parlers kabyles le «levain » (Dallet, p. 629), comme si l'élément servant à faire lever la pâte en était «l'ossature, la charpente interne ».

11 En anthropologie sociale, chez les Berbères marocains, le terme signifie «fraction de tribu» (Rif: Renisio, p. 360 ; Tamazight : Taïfi, p. 207-8 ; voir aussi Jamous 1981/2003), composante de afus*, «la main »*, qui lui désigne la tribu*; iłəss/ixss s'intégre ainsi dans un système symbolique assez cohérent où l'organisation sociale est dénommée/ organisée en analogie avec le corps humain (tête, main, paume, doigt, ventre, dos...), et dont on retrouve les traces chez tous les Berbères, (cf. notices A83 «afus », EB II ; M16 «Main », EB XXX). parenté :

Llan yəysan gar-asən : il y a des os entre eux = « ils ont des liens de parenté » (Dallet 1982, p. 629).

L'importance symbolique des os est telle que le pluriel iłsan réfère souvent à l'individu lui-même, à son essence d'être humain :

Kabyle : yəssarəd i ysan-is = il a lavé ses os = « il s'est lavé », dans le sens de « il s'est purifié, il s'est régénéré par un bain purificateur, salvateur». L'expression s'emploiera souvent en contexte religieux (pèlerinage notamment; cf. plusieurs autres exemples dans Dallet, p.629). Un emploi analogue est relevé pour la

Encyclopédie berbère, 36 | 2013 
tamazight du Maroc central par Taïfi (issird iðsan-nns, il a lavé ses os = « il a pris un bain », p. 207).

Cette identification des os à la personne elle-même est confirmée, à l'autre bout du monde berbère, par les usages du touareg méridional (Iwellemmeden*, Ayr*) :

yErza e eăs-net, il brisé os-son = « il l'a insulté ».

Iðəss/iłsan peut même remplacer le pan-berbère iman, "personne, âme, être humain », même dans les contextes où iman est quasiment grammaticalisé :

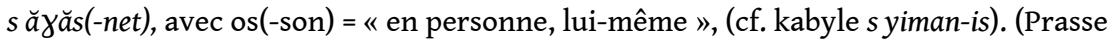

2002, p. 305 ; plusieurs exemples).

Enfin, autre trace de l'importance socioculturelle de l'os/iyəss: sa présence dans des formules de serments très anciennes, dans lesquelles on jure en évoquant les « os de ses ancêtres » :

syizsan $n$ baba... ! « Par les os de mon père... ! "

$s$ wuzu n yizsan $n$ baba... ! « Que les os de mon père soient détruits (par le feu, si)... ».

\section{(notations personnelles).}

On sera tenté d'y voir la trace ténue des pratiques funéraires protohistoriques, notamment celles du décharnement-décarnisation des cadavres, de la pratique fréquente des inhumations secondaires et de la conservation des os des défunts dans des vases funéraires comme à Tiddis*, peut-être même dans la demeure des vivants comme dans de nombreuses régions du monde (cf. Camps 1961, p. 481 à 501). On peut même se demander si les injures du français des "Pieds-noirs" du genre «La mort de tes os!» n'en sont pas un lointain écho car l'on sait que les usages linguistiques des Français d'Algérie ont été profondément influencés par les langues locales, principalement l'arabe dialectal, qui lui-même est truffé d'interférences du substrat/ adstrat berbère.

\section{BIBLIOGRAPHIE}

CAMPS G., Monuments et rites funéraires protohistoriques (Aux origines de la Berbérie), Paris, AMG, 1961.

BIARNAY S., Étude sur les dialectes berbères du Rif Paris, 1917.

CALASSANTI-MOTYLINSKI A. (de), Le Djebel Nefousa : transcription, traduction française et notes, avec une étude grammaticale, Paris, Leroux, 1898.

DALLET J.-M., Dictionnaire kabyle-français, Paris, SELAF/Peeters, 1982.

DELHEURE J., Dictionnaire mozabite-français, Paris, SELAF/Peeters, 1984.

DELHEURE J., Dictionnaire ouargli-français, Paris, SELAF/Peeters, 1987.

DESTAING E., Vocabulaire français-berbère (Etude sur la tachelhit du Soûs), Paris, Leroux, 1938.

FOUCAULD Ch. de, Dictionnaire touareg-français, 1951-2. 
HUYGHE R.P. : Dictionnaire français-chaouia, Alger, 1906.

JAMOUS R., Honneur et Baraka, les structures sociales traditionnelles dans le Rif Paris. MSH/Cambridge University Press 2003 (1981).

LANFRY, Ghadames II. Glossaire, Alger, FDB/Le Fichier Périodique, 1973.

LAOUST E., Etude sur le dialecte berbère du Chenou..., Paris, Leroux, 1912.

LAOUST E., Mots et choses berbères, Paris, Challamel, 1920 (Rabat, SMER, 1983).

G. MERCIER, Vocabulaire et textes berbères dans le dialecte des Aït Izdeg, Rabat, René Céré, 1937.

PRASSE K.-G., Manuel de grammaire touarègue (tahaggart), Copenhague, Akademisk Forlag, 1974 : IV$\mathrm{V}$, Nom.

PRASSE K.-G. et alii, Dictionnaire français-touareg, Museum Tuscululanum Press/University of Copenhague, 2003.

RENISIO A., Etude sur les dialectes berbères des Beni Iznassen, du Rif..., Paris, 1932.

TAÏFI M., Dictionnaire tamazight-français (parlers du Maroc central), Paris, L’Harmattan, 1991.

TAINE-CHEIKH C., Dictionnaire zénaga-français, Rüdiger Köppe Verlag (Berber Sudies, 20), Köln, 2008.

\section{NOTES}

1. Il existe d'autres dénominations de l'os (par ex., tamazight du sud-est : aqzzuy, aqešbab, aqežmiž, aqžžiž) mais elles sont à la fois très locales et manifestement expressives, donc secondaires.

2. Prasse (2002, p. 305) pose un /h/ (d'où *ySH), conformément à sa théorie reconstructive qui propose de restituer des laryngales dans de nombreuses formes berbères réduites. Ce point mérite débat, mais en l'absence d'une théorie comparative globale berbère/chamito-sémitique, on doit considérer toutes ces reconstructions comme purement formelles et sans véritable réalité phonétique concrète.

\section{INDEX}

Mots-clés : Ethnolinguistique, linguistique 